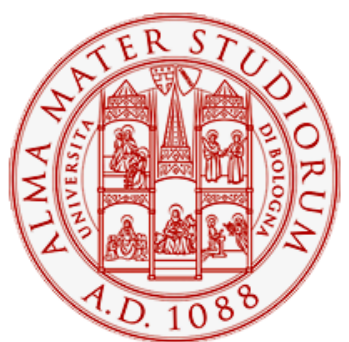

Alma Mater Studiorum - Università di Bologna DEPARTMENT OF ECONOMICS

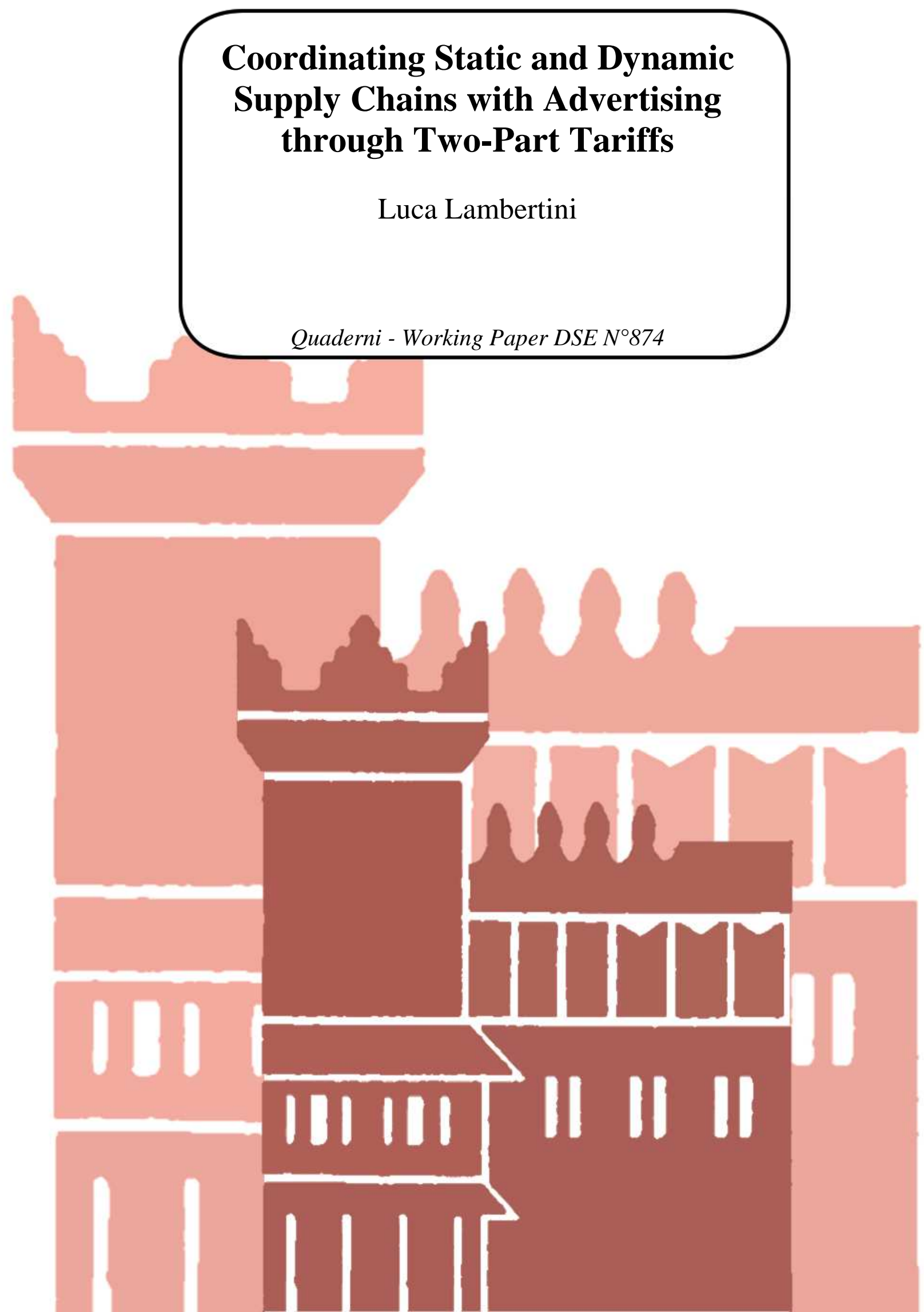




\title{
Coordinating Static and Dynamic Supply Chains with Advertising through Two-Part Tariffs ${ }^{1}$
}

\author{
Luca Lambertini \\ Department of Economics, University of Bologna \\ Strada Maggiore 45, 40125 Bologna, Italy \\ luca.lambertini@unibo.it
}

March 24, 2013

${ }^{1}$ I would like to thank Roberto Cellini and Arsen Palestini for insightful comments and discussion. The usual disclaimer applies. 


\begin{abstract}
Zaccour (2008) investigates the behaviour of a marketing channel where firms invest in advertising to increase brand equity, showing that an exogenous twopart tariff cannot be used to replicate the vertically integrated monopolist's performance. I revisit the same model proving the existence of a multiplicity of franchising contracts taht can do the job. In particular, I set out by illustrating an optimal two-part tariff specified as a linear function of the upstream firm's advertising effort, performing this task both in the static and in the dynamic game. then, I show that an analogous result emerges (i) in the static game by writing the fixed component of the two-part tariff as a non-linear function of the manufacturer's advertising effort; and (ii) by using a contract which is linear in the brand equity, in the dynamic case.
\end{abstract}

Keywords: marketing channel, vertical relations, vertical integration, advertising

JEL Codes: L21, M31, M37 


\section{Introduction}

The analysis of marketing channel behaviour is a core issue which has remained at the top of the research agenda in this field for at least thirty years, following the seminal contribution by Jeuland and Shugan (1983) and has also attracted a lot of attention in the literature on the theory of the firm belonging to the companion field of industrial economics (since Williamson, 1971; and Klein et al., 1978). A growing literature has progressively expanded the baseline model to account for the effects of (i) competition among retailers (Ingene and Parry, 1995); (ii) a dominant firm's position (Riordan, 1998; Raju and Zhang, 2005; Chen and Xiao, 2009); (iii) stochastic demand functions (Lariviere, 1999); (iv) remanufacturing (Savaskan et al., 2004); and (v) advertising (Yue et al., 2006; Zaccour, 2008).

Perhaps the most important aspect of this debate is how to specify the franchising contract, having in mind the objective of replicating in full the performance of a vertically integrated firm. While this task is not problematic if the only variable involved is price (or quantity), it becomes somewhat more intriguing when investments also enter the picture, and even more so if the model takes the form of a dynamic game. Indeed, it all boils down to specifying the equilibrium set of contracts, including - if possible - all the relevant features of the vertical relation taking place along the supply chain (Klein et al., 1978; Zusman and Etgar, 1981; Grout, 1984; Cachon and Lariviere, 2005, inter alia). May one write an efficient set of contracts safeguarding the firms' strategic incentives all along the marketing channel? Or, is it possible to write a franchising contract preventing the well known holdup problem (that is, the arising of ex post opportunistic behavior) usually 
associated with asset specifity? This is a way of telling this story revealing its proximity or analogy with well known phenomena deeply investigated in the theory of vertical integration when some form of investment is at stake (see Williamson, 1975; Klein et al., 1978; Grout, 1984; Joskow, 1985, 1987, 2005; Grossman and Hart, 1986; Hart and Moore, 1990, among many others).

The present paper nests into this line of research, being directly connected to Zaccour (2008), where it is argued that a two-part tariff allowing the marketing channel to attain the collectively optimal solution as an equilibrium of a decentralised game does not exist. This negative result is claimed in a model in which two vertically related firms invest in an advertising campaign aimed at increasing the goodwill effect (or, the brand equity), and the static and dynamic versions of the problem are both investigated. Using the same setup, I prove the existence of a multiplicity of optimal two-part tariffs driving the channel to the efficient outcome. First, I propose a tariff whose fixed component is a linear function of the upstream firm's advertising effort. If such a tariff is used in the franchising contract, the upstream firm can drive the supply chain to replicate the profits as well as the price and advertising strategies of a vertically integrated monopolist (and can appropriate the entire channel profits). I also show that the same result obtains if the upstream firm commits to its own optimal share of the advertising campaign, provided that this effort be specified in terms of the demand and intertemporal parameters of the model.

Additionally, I show that the efficient outcome can also be achieved by specifying the fixed fee appearing in the two-part tariff as a non-linear function of the manufacturer's investment, in two different but ultimately equiv- 
alent ways (in the static model). Finally, applying what is a well known solution method in hierarchical differential games, I also illustrate the possibility of reaching the efficient outcome by defining the fixed fee as a linear function of the state variable, i.e., the brand equity (in the dynamic model).

The remainder of the paper is organised as follows. Section 1 contains a short sketch of the well known optimal two-part tariff when only market variables are involved. The static version of the model is investigated in Section 2, while the dynamic game (including the commitment case) is in Section 3. The alternative solutions of the static and dynamic models are laid out in Section 4. Concluding comments are in Section 5.

\section{The simplest two-part tariff model}

As a sort of incipit, this short paragraph briefly summarises the efficient use of a two part tariff as illustrated in Jeuland and Shugan (1983, 1988a,b) and Moorthy (1987). Consider a vertical relation (a channel) between a manufacturer, $M$, and a retailer, $R$. Assume the manufacturer has a constant returns to scale technology with a unit production $\operatorname{cost} c>0$, and no investment in $\mathrm{R} \& \mathrm{D}$ or advertising is carried out by either firm. The market demand function is $Q=\beta-\alpha p$, and the unit price paid by the retailer to the manufacturer for each unit of the good is $w$. In such a case, the vertically integrated firm would attain full monopoly profits $\pi_{m}=(\beta-\alpha c)^{2} /(4 \alpha)$, and the standard view holds that vertically separated channel can replicate it by adopting a two part tariff whereby $w=c+k / Q$, or equivalently that each unit is sold by the manufacturer at marginal cost $c$ but the retailer has to pay a fixed fee $k$ 
as well, so that the two-part tariff is in fact written as $T P T=c Q+k, k$ being the fixed component. If the latter is indeed set at the full monopoly profit level, then in equilibrium the retailer obtains $\pi_{R}=(\beta-\alpha c)^{2} /(4 \alpha)-k=0$ and the manufacturer obtains $\pi_{M}=k=(\beta-\alpha c)^{2} /(4 \alpha)$. As a result, the overall channel profits are the same as under vertical integration. This consolidated conclusion has been incorporated into the standard literature on vertical relations and supply chain coordination (see, e.g., Tirole, 1988, ch. 4; Cachon, 2003; and Ingene and Parry, 2004, among many others).

\section{The static problem}

The baseline model is the same as in Zaccour (2008, p. 1234). There exists a marketing channel in which two firms linked by a vertical relation may invest in advertising to increase goodwill, so that market demand writes:

$$
Q=\beta+a_{M}+a_{R}-\alpha p
$$

where $Q$ is the output level, $p$ is market price and $\alpha_{M}$ and $\alpha_{R}$ are the advertising efforts of the manufacturer and the retailer, respectively. Each advertising campaign involves a quadratic cost $C_{i}=a_{i}^{2} / 2, i=M, R$, so that the two firms' profit functions are:

$$
\pi_{M}=(w-c) Q-\frac{a_{M}^{2}}{2} ; \pi_{R}=(p-w) Q-\frac{a_{R}^{2}}{2},
$$

while the profit function of the vertically integrated firm is:

$$
\pi_{V I}=(p-c) Q-\frac{a_{M}^{2}}{2}-\frac{a_{R}^{2}}{2} .
$$


In $(2-3), c \in(0, \beta)$ is the constant marginal production cost, and $w$ is the unit price at which the manufacturer sells the product to the retailer. Demand parameters $\alpha$ and $\beta$ are both positive, with $\alpha>1$ and $\beta>\alpha c$.

Consider first the vertically integrated solution. From the system of first order conditions (FOCs):

$$
\begin{aligned}
\frac{\partial \pi_{V I}}{\partial p} & =\beta+a_{M}+a_{R}-\alpha(2 p-c)=0 \\
\frac{\partial \pi_{V I}}{\partial a_{M}} & =p-c-a_{M}=0 \\
\frac{\partial \pi_{V I}}{\partial a_{R}} & =p-c-a_{R}=0
\end{aligned}
$$

one gets the optimal triple

$$
p^{*}=\frac{\beta+c(\alpha-2)}{2(\alpha-1)} ; a_{M}^{*}=a_{R}^{*}=\frac{\beta-\alpha c}{2(\alpha-1)},
$$

which are admissible if demand and cost parameters $\{\alpha, \beta, c\}$ satisfy the aforementioned conditions (cf. Zaccour, 2008, p. 1235). The corresponding equilibrium output and profits are:

$$
Q^{*}=\frac{\alpha(\beta-\alpha c)}{2(\alpha-1)} ; \pi_{V I}^{*}=\frac{(\beta-\alpha c)^{2}}{4(\alpha-1)} .
$$

Now, what if the vertically separated firms try to replicate the performance of the vertically integrated one by resorting to a two-part tariff defined as above, i.e., with an exogenously given fixed fee $k$ ? The result is that, by doing so, any incentive for the manufacturer to carry out its advertising campaign just disappears altogether - a clearcut example of the aforementioned hold-up problem. To see this, one proceeds by backward induction, maximising the retailer's profits

$$
\pi_{R}=(p-w)\left(\beta+a_{M}+a_{R}-\alpha p\right)-\frac{a_{R}^{2}}{2}-k
$$


w.r.t. $p$ and $a_{R}$, to obtain (superscript $T$ stands for two-part tariff):

$$
p^{T}=\frac{\beta+c(\alpha-1)+a_{M}}{2 \alpha-1} ; a_{R}^{T}=\frac{\beta-\alpha c+a_{M}}{2 \alpha-1}
$$

that can be plugged into $\pi_{M}$ together with $w=c$ to verify that the manufacturer's profit function becomes $\pi_{M}=k-a_{M}^{2} / 2$, entailing that the optimal advertising effort of the upstream firm is nil. Indeed, it I am about to illustrate that there is more to it, as there exists a way of specifying the fixed component of the two-part tariff that allows the marketing channel to exactly replicate the profit performance as well as the output and advertising investment of the vertically integrated monopolist.

To see this, write $k=x+y a_{M}$, that is, specify the fixed fee as a function of the manufacturer's advertising effort. In such a way, the fee remains fixed in that it is not a function of output, but allows one to rewrite the upstream firm's profit function as follows:

$$
\pi_{M}=x+y a_{M}-\frac{a_{M}^{2}}{2}
$$

which yields the following FOC:

$$
\frac{\partial \pi_{M}}{\partial a_{M}}=y-a_{M}=0
$$

and therefore $a_{M}^{T}=y$, which obviously requires $y>0$. If so, then the total channel advertising effort amounts to

$$
a_{M}^{T}+a_{R}^{T}=\frac{\alpha(2 y-c)+\beta}{2 \alpha-1}
$$

with $a_{M}^{T}+a_{R}^{T}=a_{M}^{*}+a_{R}^{*}$ in correspondence of $y=(\beta-c \alpha) /[2(\alpha-1)]=$ $a_{M}^{*}=a_{R}^{*}$. This exercise proves: 
Lemma 1 If $y=(\beta-c \alpha) /[2(\alpha-1)]$, total advertising investment and its single components along the marketing channel replicate the behaviour of the vertically integrated firm.

There remains to assess the profit performance and the distribution of such profits in correspondence of this particular specification of the two-part tariff. The sum of retailer's and manufacturer's profits is:

$$
\pi_{M}^{T}+\pi_{R}^{T}=\pi_{V I}^{*}=\frac{(\beta-\alpha c)^{2}}{4(\alpha-1)}
$$

while the retailer's profits are

$$
\pi_{R}^{T}=\frac{(\beta-\alpha c)^{2}(2 \alpha-3)-8 x(\alpha-1)^{2}}{8(\alpha-1)^{2}} .
$$

Consequently, setting $x=(\beta-\alpha c)^{2}(2 \alpha-3) /\left[8(\alpha-1)^{2}\right]$, the manufacturer can appropriate the entire channel profits in correspondence of the efficient solution which would be attained under vertical integration. This amounts to saying

Proposition 2 In correspondence of $y=(\beta-c \alpha) /[2(\alpha-1)]$ and $x=$ $(\beta-\alpha c)^{2}(2 \alpha-3) /\left[8(\alpha-1)^{2}\right]$, the channel's performance is the same as the vertically integrated monopolist's, and the entire profits accrue to the upstream firm.

In other words, this contract entails setting

$$
k=x+y a_{R}=\frac{(\beta-\alpha c)^{2}(2 \alpha-1)}{8(\alpha-1)^{2}}>0 .
$$

As an ancillary but relevant remark, it is worth stressing that (12-13) also imply the following: 
Corollary 3 There exist infinitely many optimal contracts allowing the supply chain to perform efficiently, summarised by any pair $y=(\beta-c \alpha) /[2(\alpha-1)]$ and $x \in\left\{x \mid \pi_{R}^{T} \geq 0\right\}$.

Needless to say, the choice of the specific contract driving to zero the retailer's profits is the most advantageous for the manufacturer, which might instead leave the retailer with some positive (but arbitrarily small) profit $\varepsilon$ by setting $\widehat{x}=x-\varepsilon$.

\section{The dynamic problem}

Again, the model is the same as in Zaccour (2008, pp. 1236-37). The time horizon is infinite, with $t \in[0, \infty)$, and both firms share the same intertemporal preferences summarised by the discount rate $\rho>0$. Advertising controls are $a_{M}(t)$ and $a_{R}(t)$, entailing instantaneous costs $C_{i}\left[a_{i}(t)\right]=a_{i}^{2} / 2$, $i=M, R$. The production of the final good involves a constant marginal cost c. The goodwill (or brand equity) dynamics is

$$
\dot{B}(t)=a_{M}(t)+a_{R}(t)-\delta B(t)
$$

with the initial condition $B(0)=B_{0}>0$; parameter $\delta>0$ is the constant decay rate. The instantaneous demand function is $Q(t)=\mu+B(t)-\alpha p(t)$, with $\alpha>1 /[\delta(\delta+\rho)]$ and $\mu>\alpha c .^{1}$

The equilibrium solution for the vertically integrated channel is described in Zaccour (2008, Proposition 1, p. 1237), and is briefly summarised here

\footnotetext{
${ }^{1}$ The condition $\alpha>1 /[\delta(\delta+\rho)]$ is required for stability, while the second condition on $\mu$ ensures that the brand equity be positive in equilibrium.
} 
(in the remainder, the explicit indication of the time argument is omitted for brevity). Adopting the quadratic value function $V(B)=\varphi_{1} B^{2} / 2+\varphi_{2} B+\varphi_{3}$, one obtains the vector of optimal controls:

$$
\begin{gathered}
p^{V I}(B)=\frac{\mu+B+\alpha c}{2 \alpha} \\
a_{i}^{V I}(B)=\varphi_{1} B+\varphi_{2}, i=M, R
\end{gathered}
$$

with

$$
\begin{gathered}
\varphi_{1}=\frac{\alpha(2 \delta+\rho)-\sqrt{\alpha\left[\alpha(2 \delta+\rho)^{2}-4\right]}}{\varphi_{2}=\frac{\mu \alpha \alpha c}{2 \alpha\left(2 \varphi_{1}-\delta-\rho\right)}} \\
\varphi_{3}=\frac{4 \alpha \varphi_{2}^{2}-(\mu-\alpha c)^{2}}{4 \alpha \rho}
\end{gathered}
$$

It is worth noting that in Zaccour $\left(2008\right.$, p. 1237) the expression of $\varphi_{2}$ does not contain $\alpha$ at the denominator. As a result, the equilibrium level of the brand equity, which is

$$
B^{V I}=\frac{\mu-\alpha c}{\alpha \delta(\delta-\rho)-1}
$$

in Zaccour (2008, pp. 1237-38) is multiplied by $\alpha$. This has no particular consequences on the second step of Zaccour's analysis, portrayed in his Proposition 2 (Zaccour, 2008, p. 1238), which shows that, when the two firms are independent units, the wholesale two-part tariff $w=c+k / Q$ cannot allow the marketing channel to replicate the performance of the vertically integrated supply chain, the reason being that $\pi_{M}=k-a_{M}^{2} / 2$ says that it is efficient for the manufacturer not to invest at all in advertising. Moreover, neither a precommitment on the part of the manufacturer to carry out his own share of advertising, $a_{R}=\varphi_{1} B+\varphi_{2}$, can do the job (Zaccour, 2008, Proposition 3, p. 1238) All this indeed holds true as long as $k$ is exogenous. 
I am about to show that taking the same route as in the above static model, this conclusion flips over altogether.

For the moment, write the two-part tariff as $w=c+k / Q$. Posing the retailer's value function as $V_{R}(B)=\theta_{1} B^{2} / 2+\theta_{2} B+\theta_{3}$, the retailers' Bellman equation is

$$
\begin{aligned}
\rho V_{R}(B)= & \max _{p, a_{R}}\left[\left(p-\frac{k}{B+\mu-\alpha p}\right)(B+\mu-\alpha p)\right. \\
& \left.-\frac{a_{R}^{2}}{2}+\left(\theta_{1} B+\theta_{2}\right)\left(a_{M}+a_{R}-\delta B\right)\right]
\end{aligned}
$$

which generates the following optimal controls:

$$
\begin{gathered}
p^{T}(B)=\frac{\mu+B+\alpha c}{2 \alpha} \\
a_{R}^{T}=\theta_{1} B+\theta_{2} .
\end{gathered}
$$

These can be substituted back into (19), rewriting thus the latter as

$$
\begin{gathered}
\frac{\left[1+2 \alpha\left(\theta_{1}-2 \delta-\rho\right) \theta_{1}\right] B^{2}}{4 \alpha}+\frac{\left[\mu-\alpha\left(2(\delta+\rho) \theta_{2}-2\left(a_{M}+\theta_{2}\right) \theta_{1}+c\right)\right] B}{2 \alpha}+ \\
\frac{\alpha^{2} c^{2}+\mu^{2}+2 \alpha\left[\theta_{2}\left(\theta_{2}+2 a_{M}\right)-c \mu-2\left(k+\rho \theta_{3}\right)\right]}{4 \alpha}=0
\end{gathered}
$$

Solving the resulting system of three equations w.r.t. the unknown parameters $\left\{\theta_{1}, \theta_{2}, \theta_{3}\right\}$, one obtains: ${ }^{2}$

$$
\begin{gathered}
\theta_{3}=\frac{\alpha^{2} c^{2}+\mu^{2}+2 \alpha\left[\theta_{2}\left(\theta_{2}+2 a_{M}\right)-c \mu-2 k\right]}{4 \alpha \rho} \\
\theta_{2}=\frac{\mu+\alpha\left(2 \theta_{1} a_{M}-c\right)}{4 \alpha \rho} \\
\theta_{1}=\frac{\alpha(2 \delta+\rho)-\sqrt{\alpha\left[\alpha(2 \delta+\rho)^{2}-2\right]}}{2 \alpha}
\end{gathered}
$$

\footnotetext{
${ }^{2}$ The larger solution for $\theta_{1}$ is disregarded as it cannot ensure stability.
} 
Now turn to the manufacturer's Bellman equation:

$$
\rho V_{M}(B)=\max _{p, a_{R}}\left[k-\frac{a_{M}^{2}}{2}+\frac{\partial V_{M}(B)}{\partial B}\left(a_{M}+a_{R}^{T}-\delta B\right)\right]
$$

in which $a_{R}^{T}$ corresponds to the expression appearing in (20). Hence, (23) can be rewritten as

$$
\begin{gathered}
{\left[\left(a_{M}^{2}+2\left(V_{M}(B)-k\right)\right) \sqrt{\alpha\left(\alpha(2 \delta+\rho)^{2}-2\right)}+2 \frac{\partial V_{M}(B)}{\partial B}(B(2 \alpha \delta(\delta+\rho)-1)-\mu)\right.} \\
\left.+\alpha\left(\rho\left(a_{M}^{2}+2\left(\rho V_{M}(B)-k\right)\right)+2 \frac{\partial V_{M}(B)}{\partial B}\left(c-2 a_{M}(\delta+\rho)\right)\right)\right] /(24) \\
{\left[2\left(\alpha \rho+\sqrt{\alpha\left(\alpha(2 \delta+\rho)^{2}-2\right)}\right)\right]=0 .}
\end{gathered}
$$

Now, following the same procedure as in the static problem, one can set $k=v+m a_{M}$, where parameters $v$ and $m$ are to be identified appropriately in the remainder, so that the resulting FOC for the maximisation of (24) w.r.t. $a_{M}$ yields:

$$
a_{M}^{T}=\frac{m \sqrt{\alpha\left(\alpha(2 \delta+\rho)^{2}-2\right)}+\alpha\left[2 \frac{\partial V_{M}(B)}{\partial B} \delta+\rho\left(m+2 \frac{\partial V_{M}(B)}{\partial B}\right)\right]}{\alpha \rho+\sqrt{\alpha\left(\alpha(2 \delta+\rho)^{2}-2\right)}} .
$$

The next step consists in specifying the manufacturer's value function as $V_{M}(B)=\psi_{1} B^{2} / 2+\psi_{2} B+\psi_{3}$, with $\partial V_{M}(B) / \partial B=\psi_{1} B+\psi_{2}$, and then solve the system of three equations implied by (24) w.r.t. the unknown parameters $\left\{\psi_{1}, \psi_{2}, \psi_{3}\right\}$. This delivers:

$$
\psi_{1}=\psi_{2}=0, \psi_{3}=\frac{4 v+3 m^{2}}{\rho} .
$$

It is worth noting that (26) says that 'the state variable is irrelevant', and the manufacturer's problem collapses onto a quasi-static one. Before proceeding 
any further, it is worth noting that the above result implies that any credible precommitment by the manufacturer cannot be based on the parameters of its value function (for more on this aspect, see below).

Imposing stationarity on the state variable, one obtains

$$
B^{T}=\frac{\mu+\alpha[2(\delta+\rho) m-c]}{2 \alpha \delta(\delta+\rho)-1} .
$$

Moreover, the individual and total advertising efforts along the marketing channel simplify as follows:

$$
a_{M}^{T}=y ; a_{M}^{T}+a_{R}^{T}=\frac{\delta[\mu+\alpha(2(\delta+\rho) m-c)]}{2 \alpha \delta(\delta+\rho)-1}
$$

the latter being equal to $a^{V I}=\delta(\mu-\alpha c) /[\alpha \delta(\delta+\rho)-1]$ at

$$
m=\frac{\delta(\mu-\alpha c)}{2[\alpha \delta(\delta+\rho)-1]}=\frac{a^{V I}}{2}
$$

which also ensures $a_{M}^{T}=a_{R}^{T}=a^{V I} / 2$.

Concerning profits, we have that

$$
\pi_{M}^{T}+\pi_{R}^{T}=\pi^{V I}=\frac{\delta^{2}(\mu-\alpha c)^{2}\left[\alpha(\delta+\rho)^{2}-1\right]}{4[\alpha \delta(\delta+\rho)-1]^{2}}
$$

and

$$
\pi_{M}^{T}=\frac{\delta^{2}(\mu-\alpha c)^{2}}{8[\alpha \delta(\delta+\rho)-1]^{2}}+v=\pi^{V I}
$$

in $v=\delta^{2}(\mu-\alpha c)^{2}\left[2 \alpha(\delta+\rho)^{2}-3\right] /\left[8(\alpha \delta(\delta+\rho)-1)^{2}\right]$.

The foregoing discussion can be summarised in

Proposition 4 Adopting the two-part tariff $w+k / Q$, with $k=v+m a_{M}$ and

$$
v=\frac{\delta^{2}(\mu-\alpha c)^{2}\left[2 \alpha(\delta+\rho)^{2}-3\right]}{8[\alpha \delta(\delta+\rho)-1]^{2}} ; m=\frac{\delta(\mu-\alpha c)}{2[\alpha \delta(\delta+\rho)-1]}=\frac{a^{V I}}{2},
$$


the manufacturer (i) induces the marketing channel to replicate the advertising and profit performance of the vertically integrated monopolist and (ii) appropriates the whole profits generated by the supply chain.

The bottom line of the analysis carried out in both the static and the dynamic settings is that the efficient two-part tariff must be defined in terms of the manufacturer's advertising effort, in such a way as to restore the incentive for the upstream firm to exactly replicate the advertising campaign that the upstream division of a vertically integrated monopolist would carry out. In this sense, the fixed part of the tariff administers the appropriate stimulus to the manufacturer, as illustrated by (25). Relatedly, it is also worth stressing that this outcome and the franchising contract which generates it are strongly time consistent - not only because this is a feedback equilibrium (which is the technical reason), but because it is in the manufacturer's best interest to perform his share of the optimal advertising campaign and then use the coefficients of the two-part tariff to appropriate monopoly profits: any deviations by the manufacturer from this line of behaviour would jeopardise its profits.

As a last step of this part of the analysis, one can ask whether the static problem can be considered as a special case of the dynamic one. A quick examination of equilibrium magnitudes reveals that it is indeed so. To make the two settings directly comparable, fix $\beta=\mu$, so that the vertical intercept of the demand function, in absence of brand equity effects driven by advertising campaigns, is indeed the same. Having done that, all the equilibrium magnitudes observed in correspondence of the equilibrium of the dynamic game coincide with those characterising the equilibrium of the static game if $\delta=1$ 
and $\rho=0$ (one needs not take limits to verify this, as simple substitutions do the job). That is to say:

Corollary 5 If the goodwill instantaneous decay rate is $100 \%$ and firms do not discount future gains, then the dynamic solution of the feedback game collapses onto the solution of the static game.

This conclusion is strongly reminiscent of qualitatively analogous results attained in applications of differential games to completely different issues (such as the sticky price game dating back to Simaan and Takayama, 1978, and further investigated by Fershtman and Kamien, 1987; Dockner, 1988; and Cellini and Lambertini, 2004, 2007).

\subsection{The commitment solution}

Zaccour (2008, p. 1236) shows that, in the static setup, a commitment to perform its own share of the advertising campaign on the part of the manufacturer - alongside with the two-part tariff - allows the supply chain to replicate the performance of a vertically integrated monopolist. There remains to investigate whether the same result obtains in the dynamic setting, if the manufacturer commits to its own share of advertising investment,

$$
\bar{a}_{M}=a_{M}^{V I}=\frac{\delta(\mu-\alpha c)}{2[\alpha \delta(\delta+\rho)-1]}
$$

accompanied by the two-art tariff $w=c+k / Q$ (the bar on $a_{M}$ indicates the presence of a commitment) Note that (32) defines the manufacturer's commitment in terms of the model parameters, instead of the parameters of the manufacturer's value function $\left\{\psi_{1}, \psi_{2}\right\}$ and the state variable $B$ as in 
Zaccour (2008, p. 1238, proof of Proposition 3). The reason is that, as shown above, $\psi_{1}=\psi_{2}=0$, and therefore writing the contract in this way could not work (as stressed by Zaccour): in fact, the above argument illustrates that the requirement that $\left\{\psi_{1}, \psi_{2}\right\}$ coincide with the parameters characterising the vertically integrated solution is misleading as the optimal two-part tariff turns the manufacturer's problem into a quasi-static one. Therefore, one has instead to specify $a_{M}$ as in (32) and then check whether the solution of the retailer's Bellman equation:

$$
\begin{aligned}
\rho V_{R}(B)= & \max _{p, a_{R}}\left[\left(p-\frac{k}{B+\mu-\alpha p}\right)(B+\mu-\alpha p)\right. \\
& \left.-\frac{a_{R}^{2}}{2}+\left(\theta_{1} B+\theta_{2}\right)\left(\frac{\delta(\mu-\alpha c)}{2(\alpha \delta(\delta+\rho)-1)}+a_{R}-\delta B\right)\right]
\end{aligned}
$$

is the same as above. Indeed, the FOCs taken on (33) generate (20). This is obvious, as the above expression is additively separable in the two firms' advertising efforts, and therefore the manufacturer's behaviour does not enter the retailer's optimality conditions. Solving the resulting system of three equations w.r.t. the unknown parameters $\left\{\theta_{1}, \theta_{2}, \theta_{3}\right\}$, one obtains:

$$
\begin{aligned}
\theta_{3}= & {\left[\left(\alpha^{2} c^{2}+\mu^{2}-2 \alpha(2 k+c \mu)\right)(\alpha \delta(\delta+\rho)-1)\right.} \\
& \left.+2 \alpha \theta_{2}\left(\theta_{2}(\alpha \delta(\delta+\rho)-1)+\delta(\mu-\alpha c)\right)\right] / \\
& {[4 \alpha \rho(\alpha \delta(\delta+\rho)-1)] }
\end{aligned}
$$

and

$$
\begin{gathered}
\theta_{2}=\frac{(\mu-\alpha c)\left[\alpha \delta\left(\delta+\rho+\theta_{1}\right)-1\right]}{2 \alpha(\alpha \delta(\delta+\rho)-1)\left(\delta+\rho-\theta_{1}\right)} \\
\theta_{1}=\frac{\alpha(2 \delta+\rho)-\sqrt{\alpha\left[\alpha(2 \delta+\rho)^{2}-2\right]}}{2 \alpha}
\end{gathered}
$$

This yields $\bar{a}_{R}=a_{M}^{T}=a_{M}^{V I}=a^{V I} / 2$.Then, solving $\dot{B}=0$, we have the same solution as in (18). The final exercise consists in verifying that total channel 
profits do coincide with $\pi^{V I}$ in (30). The answer is positive, as

$$
\bar{\pi}_{M}+\bar{\pi}_{R}=\pi^{V I}=\frac{\delta^{2}(\mu-\alpha c)^{2}\left[\alpha(\delta+\rho)^{2}-1\right]}{4[\alpha \delta(\delta+\rho)-1]^{2}}
$$

with

$$
\bar{\pi}_{M}=k-\frac{\delta^{2}(\mu-\alpha c)^{2}}{8[\alpha \delta(\delta+\rho)-1]^{2}}
$$

so that the manufacturer can grasp the whole monopoly profits by setting

$$
k=\frac{\delta^{2}(\mu-\alpha c)^{2}\left[2 \alpha(\delta+\rho)^{2}-1\right]}{8[\alpha \delta(\delta+\rho)-1]^{2}} .
$$

This proves:

Proposition 6 The precommitment solution

$$
\bar{a}_{M}=\frac{\delta(\mu-\alpha c)}{2[\alpha \delta(\delta+\rho)-1]} ; w=c+k / Q ; k=\frac{\delta^{2}(\mu-\alpha c)^{2}\left[2 \alpha(\delta+\rho)^{2}-1\right]}{8[\alpha \delta(\delta+\rho)-1]^{2}}
$$

allows the marketing channel to replicate the performance of the vertically integrated firm.

\section{Alternative solutions}

Here I will pursue some alternative routes, the first two to deal with the static model, the third with the dynamic one, respectively. In the static case, it can be shown that (i) if the fixed fee is explicitly designed to transfer the full profits to the manufacturer, then the investment incentive of the latter is fully restored; and (ii) the solution envisaged for the dynamic case is indebted with what is by now a standard approach to generating Markovian equilibria in Stackelberg differential games, as it consists in specifying the leader's strategy as a linear function of the state variable (see Dockner et al., 2000, pp. 134-41). 


\subsection{The static problem: route I}

The first possibility consists in deriving the optimal price and advertising effort of the retailer, which coincide with (8), and then in simplifying the retailer's profits with $w=c$, given a generic level of the manufacturer's advertising effort $a_{M}$ :

$$
\pi_{R}=\frac{\left(\beta+a_{M}-\alpha c\right)^{2}}{2(2 \alpha-1)}-k
$$

Finally, one has to impose

$$
k^{T}=\frac{\left(\beta+a_{M}-\alpha c\right)^{2}}{2(2 \alpha-1)}
$$

which is tantamount to saying that the fixed fee operates a complete transfer of profits from the downstream firm to the upstream one. ${ }^{3}$

Moving upwards, the manufacturer has to maximise the following profit function:

$$
\pi_{M}=\frac{\left(\beta+a_{M}-\alpha c\right)^{2}}{2(2 \alpha-1)}-\frac{a_{M}^{2}}{2}
$$

with $\partial \pi_{M} / \partial a_{M}=0$ at

$$
a_{M}=\frac{\beta-\alpha c}{2(\alpha-1)}=a_{M}^{*}
$$

All of the resulting expression (the retailer's advertising and price, as well as channel profits) coincide with those associated with the vertically integrated equilibrium. The foregoing discussion can be summarised in

\footnotetext{
${ }^{3} \mathrm{~A}$ softer version of the same scheme would be to set

$$
k=\frac{\left(\beta+a_{M}-\alpha c\right)^{2}}{2(2 \alpha-1)}-\varepsilon
$$

with $\varepsilon \in\left(0,\left(\beta+a_{M}-\alpha c\right)^{2} /[2(2 \alpha-1)]\right)$.
} 
Proposition 7 The two-part tariff identified by

$$
w=c \text { and } k^{T}=\left(\beta+a_{M}-\alpha c\right)^{2} /[2(2 \alpha-1)]
$$

solves the problem of coordinating the vertical channel, leading the latter to replicate the performance of the vertically integrated firm. The entire channel profits accrue to the upstream firm.

It is worth stressing that this solution is close in spirit to that usually adopted in vertical relation models with franchising, without investments of any sort, as already illustrated in Section 2. The only difference is that here the fixed fee has to take explicitly into account the manufacturer's effort $a_{M}$.

\subsection{The static problem: route II}

Alternatively, exploiting an analogy with the theory of potential games (Slade, 1994; Monderer and Shapley, 1996), ${ }^{4}$ one may reformulate the research question as follows: may the marketing channel coordination game involving advertising efforts admit a potential, and may the latter reproduce with the profits of a vertically integrated monopolist, through the use of an appropriate two-part tariff? More precisely, is it possible to identify a two-part tariff such that, indeed, the potential function replicating the equilibrium between two vertically related but independent firms also replicates the objective function of the vertically integrated firm, and therefore equilibrium profits coincide across the two cases? In this respect, we already know that

\footnotetext{
${ }^{4}$ This branch of game theory has been largely developed for static games, although some preliminary results are available also for differential games. See Dragone et al. (2008, 2012).
} 
the Stackelberg game involving only a price or quantity choice does admit a potential via the well known two-part tariff outlined at the outset, and that this potential corresponds to the vertically integrated solution. The issue is now whether the present problem, enriched by the presence of advertising efforts, delivers an analogous outcome or not.

To answer this question, observe the first order condition of the vertically integrated firm w.r.t. $a_{M}$, given the price $p^{T}$ in (8):

$$
p^{T}-c-a_{M}=\frac{\beta-c \alpha-2 a_{M}(b-1)}{2 \alpha-1} .
$$

By imposing $p^{T}-c-a_{M}=0$, one gets precisely the same advertising effort associated with the vertically integrated solution (5). Accordingly, take the indefinite integral

$$
\int \frac{\beta-c \alpha-2 a_{M}(\alpha-1)}{2 \alpha-1} d a_{M}=\frac{a_{M}\left[\beta-c \alpha-a_{M}(\alpha-1)\right]}{2 \alpha-1}+C \equiv \Xi\left(a_{M}\right)
$$

where $C$ is the integration constant, which can be set equal to zero for simplicity and without further loss of generality. Then, solve

$$
\Xi\left(a_{M}\right)-\pi_{M}=\frac{a_{M}\left[\beta-c \alpha-a_{M}(\alpha-1)\right]}{2 \alpha-1}-k+\frac{a_{M}^{2}}{2}=0
$$

w.r.t. $k$, to obtain

$$
k^{T}=\frac{a_{M}\left[a_{M}+2(\beta-c \alpha)\right]}{2(2 \alpha-1)} .
$$

This amounts to imposing that the manufacturer profits, $\pi_{M}=k-a_{M R}^{2} / 2$, be equal to a function $\Xi\left(a_{M}\right)$ of its advertising effort obtained by integrating w.r.t. $a_{M}$ the first order condition of the vertically integrated monopolist w.r.t. the same variable. It is worth noting that the above procedure yields 
a specification of the fixed fee which is non-linear in the manufacturer's advertising effort.

Once $k$ has been defined as in (46), the resulting profit function of the manufacturer, $\pi_{M}\left(k^{T}\right)$, produces the FOC:

$$
\frac{\partial \pi_{M}}{\partial a_{M}}=\frac{2(1-\alpha) a_{M}+\beta-c \alpha}{2 \alpha-1}=0
$$

whereby the optimal advertising effort is $a_{M}^{T}=a_{M}^{*}$, i.e., the same as under the vertically integrated solution. The same holds for the equilibrium consumer price and quantity and the advertising effort of the retailer. The equilibrium profits are:

$$
\pi_{M}^{T}=\frac{(\beta-c \alpha)^{2}}{4(2 \alpha-1)(\alpha-1)} ; \pi_{R}^{T}=\frac{(\beta-c \alpha)^{2}}{2(2 \alpha-1)},
$$

with $\pi_{M}^{T}+\pi_{R}^{T}=\pi_{V I}^{*}$ and $\pi_{R}^{T}>\pi_{M}^{T}$ for all $\alpha>3 / 2$. That is:

Proposition 8 The two-part tariff identified by

$$
w=c \text { and } k^{T}=a_{M}\left[a_{M}+2(\beta-c \alpha)\right] /[2(2 \alpha-1)]
$$

solves the problem of coordinating the vertical channel, leading the latter to replicate the performance of the vertically integrated firm. In this case, however, the retailer earns positive profits, which are larger than the manufacturer if the demand function is steep enough.

To summarise: the retailer maximises (7) w.r.t. $p$ and $a_{R}$, with $k=k^{T}$, which leaves unaffected the retailer's FOCs as $k^{T}$ is a function of $a_{M}$ only (plus obviously the demand and cost parameters). Going backwards to the first stage of the sequential game, the use of $k^{T}$ allows to reproduce the same advertising incentive for the manufacturer as in the vertically integrated case. 
Therefore, in both cases the solution to Jeuland and Shugan's problem consists in specifying the contract (that is, the two part tariff) in terms of the manufacturer effort, in particular as a quadratic function of the latter. This remains, in a sense, exogenous to the retailer's behaviour (for the reason stated above), although it is not entirely exogenous to the vertical relation as a whole, as it is conditional on the advertising investment of firm $M$. What matters is that imposing $k=k^{T}$ (i) has no influence on the retailer's FOCs, but restores the manufacturer's incentive to carry out the efficient advertising investment; and (ii) involves writing the franchising contract not in terms of a fixed fee but an endogenous one, that still poses no time inconsistency issue as it does not enter the retailer's optimality conditions.

\subsection{The dynamic setup}

This is an alternative view of the hierarchical differential game along the marketing channel. Using again $w=c+k / Q$, the analysis of the dynamic game replicates (19-22). Here, however, I pose $k=v+m B$, so that the Bellman equation of the manufacturer becomes

$$
\rho V_{M}(B)=\max _{p, a_{R}}\left[v+m B-\frac{a_{M}^{2}}{2}+\frac{\partial V_{M}(B)}{\partial B}\left(a_{M}+a_{R}^{T}-\delta B\right)\right]
$$

with $a_{R}^{T}=\theta_{1} B+\theta_{2}$. Supposing $V_{M}(B)=\psi_{1} B^{2} / 2+\psi_{2} B+\psi_{3}$, the FOC w.r.t. $a_{M}$ yields

$$
a_{M}^{T}=\frac{2 \alpha(\delta+\rho) \frac{\partial V_{M}(B)}{\partial B}(\delta+\rho)}{\alpha \rho+\sqrt{\alpha\left(\alpha(2 \delta+\rho)^{2}-2\right)}}
$$

which, substituted back into (49), gives rise to a system of three equations in the unknown parameters $\left\{\psi_{1}, \psi_{2}, \psi_{3}\right\}$. The solution of this system is the 
following triple:

$$
\begin{gathered}
\psi_{3}=\left[\alpha \psi_{2}\left(\mu \rho+\alpha\left(2 \psi_{2}(\delta+\rho)^{2}-c \rho\right)\right)+2 \alpha\left(\alpha\left(2 \delta(\delta+\rho)+\rho^{2}\right)-1\right) v+\right. \\
\left.\left(2 \alpha \rho v+\psi_{2}(\mu-c \alpha)\right) \Omega\right] /\left[2 \alpha \rho\left(\alpha\left(2 \delta(\delta+\rho)+\rho^{2}\right)-1+\rho\right) \Omega\right] \\
\text { with } \Omega \equiv \sqrt{\alpha\left(\alpha(2 \delta+\rho)^{2}-2\right)} \text {; and } \\
\qquad \psi_{2}=\frac{2 \alpha m}{\alpha \rho+\Omega} ; \psi_{1}=0
\end{gathered}
$$

so that (51-52) reveal that the use of a state-dependent rule turns the upstream firm's problem into a linear state one.

The steady state value of $B$ is then

$$
B=\frac{(\mu-c \alpha)[1-2 \alpha \delta(\delta+\rho)]^{2}+4 \alpha^{2}(\delta+\rho)^{2}\left[\alpha\left(2 \delta(\delta+\rho)+\rho^{2}\right)-1-\rho \Omega\right] m}{[2 \alpha \delta(\delta+\rho)-1]^{3}}
$$

and fixing

$$
m=\frac{\delta(\mu-c \alpha)[1-2 \alpha \delta(\delta+\rho)]^{2}}{4 \alpha(\delta+\rho)[\alpha \delta(\delta+\rho)-1]\left[\alpha\left(2 \delta(\delta+\rho)+\rho^{2}\right)-1-\rho \Omega\right]}
$$

ensures $a_{M}^{T}+a_{R}^{T}=a^{V I}, a_{M}^{T}=a_{M}^{V I}=a^{V I} / 2$ and $B^{T}=B^{V I}$, with $\pi_{M}^{T}+\pi_{R}^{T}=$ $\pi^{V I}$ as well. If the manufacturer sets

$$
v=\frac{\delta(\mu-c \alpha)^{2}\left[2\left(1+\alpha^{2} \delta(\delta+\rho)^{3}\right)-\alpha\left(5 \delta(\delta+\rho)+2 \rho^{2}\right)-2 \rho \Omega\right]}{8 \alpha(\delta+\rho)[\alpha \delta(\delta+\rho)-1]^{2}},
$$

then $\pi_{M}^{T}=\pi^{V I}$ and $\pi_{R}^{T}=0$.

The foregoing discussion can be summed up as follows:

Proposition 9 The marketing channel can replicate the outcome of a vertically integrated monopolist using a two-part tariff in which the fixed fee is linear in the stock of brand equity. 


\section{Concluding remarks}

I have illustrated the possibility for a supply chain consisting of two vertically related but independent firms of using an optimal two-part tariff to achieve efficiency, in a setting where firms are assumed to undertake costly investments to increase the brand equity or goodwill. There emerges a nonuniqueness result, as there is a multiplicity of two-part tariffs achieving the desired outcome. In the dynamic setup, such a tariff may be specified in two different forms, one being linear in the upstream firm's advertising effort, the other being linear in the state variable (the stock of goodwill). In both cases, the fee remains independent of the output level. In the static setup, efficiency can be attained through linear and non-linear contracts defined in terms of the manufacturer's effort.

Much remains to be done in order to explore the possibility of extending these results to a wider set of models, of which the present one represents a specific example. For instance, plausible routes to be pursued are (i) allowing for oligopolistic interaction either upstream or downstream (or both), leaving other essential elements of the model unmodified; (ii) exploring the applicability of similar contracts in models where R\&D for process and product innovation replace advertising efforts; and (iii) allowing for more sophisticated strategies such as market-share discounts (as in Calzolari and Denicolò, 2013). These open issues are left for future research. 


\section{References}

[1] Cachon, G.P. (2003), "Supply Chain Coordination with Contracts", in S. Graves and T. de Kok (eds), Handbook in Operation Research and Management Science: Supply Chain Management, Amsterdam, NorthHolland.

[2] Cachon, G.P. and M.A. Lariviere (2005), "Supply Chain Coordination with Revenue-Sharing Contracts: Strengths and Limitations", Management Science, 51, 30-44.

[3] Calzolari, G. and V. Denicolò, (2013), "Competition with Exclusive Contracts and Market-Share Discounts", American Economic Review, forthcoming.

[4] Cellini, R. and L. Lambertini (2004), "Dynamic Oligopoly with Sticky Prices: Closed-Loop, Feedback and Open-Loop Solutions", Journal of Dynamical and Control Systems, 10, 303-14.

[5] Cellini, R. and L. Lambertini (2007), "A Differential Oligopoly Game with Differentiated Goods and Sticky Prices", European Journal of Operational Research, 176, 1131-44.

[6] Chen, K. and T. Xiao (2009), "Demand Disruption and Coordination of the Supply Chain with a Dominant Retailer", European Journal of Operational Research, 197, 225-34. 
[7] Dockner, E. (1988), "On the Relation between Dynamic Oligopolistic Competition and Long-Run Competitive Equilibrium", European Journal of Political Economy, 4, 47-64.

[8] Dockner, E., S. Jørgensen, N.V. Long and G. Sorger (2000), Differential Games in Economics andManagement Science, Cambridge, Cambridge University Press.

[9] Dragone, D., L. Lambertini and A. Palestini (2012), "Static and Dynamic Best-Response Potential Functions for the Non-Linear Cournot Game", Optimization, 61, 1283-93.

[10] Dragone, D., L. Lambertini, G. Leitmann and A. Palestini (2008), "Hamiltonian potential functions for differential games", working paper no. 644, Department of Economics, University of Bologna.

[11] Fershtman, C. and M.I. Kamien (1987), "Dynamic Duopolistic Competition with Sticky Prices", Econometrica, 55, 1151-64.

[12] Grossman, S. and O. Hart (1986), "The Costs and Benefits of Ownership: A Theory of Vertical and Lateral Integration", Journal of Political Economy, 94, 691-719.

[13] Grout, P. (1984), "Investment and Wages in the Absence of Binding Contracts: A Nash Bargining Approach", Econometrica, 52, 449-60.

[14] Hart, O. and J. Moore (1990), "Property Rights and the Nature of the Firm", Journal of Political Economy, 98, 1119-58. 
[15] Ingene, C.A. and M.E. Parry (1995), "Channel Coordination when Retailers Compete", Marketing Science, 14, 360-77.

[16] Ingene, C.A. and M.E. Parry (2004), Mathematical Models of Distribution Channels, Boston, MA, Kluwer Academic Publishers.

[17] Jeuland, A.P. and S.M. Shugan (1983), "Managing Channel Profits", Marketing Science, 2, 239-72.

[18] Jeuland, A.P. and S.M. Shugan (1988a), "Reply To: Managing Channel Profits: Comment", Marketing Science, 7, 103-06.

[19] Jeuland, A.P. and S.M. Shugan (1988b), "Channel of Distribution Profits when Channel Members Form Conjectures", Marketing Science, 7, 202-10.

[20] Joskow, P. (1985), "Vertical Integration and Long Term Contracts: The Case of Coal-burning Electric Generating Stations", Journal of Law, Economics and Organization, 1, 33-80.

[21] Joskow, P. (1987), "Contract Duration and Relationship-Specific Investments: Empirical Evidence from Coal Markets", American Economic Review, 67, 168-85.

[22] Joskow, P. (2005), "Vertical Integration", in C. Menard and M. Shirley (eds), Handbook of New Institutional Economics, Heidelberg, Springer.

[23] Klein, B., R. Crawford and A. Alchian (1978), "Vertical Integration, Appropriable Rents, and the Competitive Contracting Process", Journal of Law and Economics, 21, 297-326. 
[24] Lariviere, M.A. (1999), "Supply Chain Contracting and Coordination with Stochastic Demand", in S. Tayur, R. Ganeshan and M. Magazine (eds), Quantitative Models for Supply Chain Management, Boston, Kluwer Academic Publishers.

[25] Monderer, D. and L.S. Shapley (1996), "Potential Games", Games and Economic Behavior, 14, 124-43.

[26] Moorthy, K.S. (1987), "Managing Channel Profits: Comment", Marketing Science, 6, 375-79.

[27] Raju, J. and Z.J. Zhang (2005), "Channel Coordination in the Presence of a Dominant Retailer", Marketing Science, 24, 254-62.

[28] Riordan, M.H. (1998), “Anticompetitive Vertical Integration by a Dominant Firm", American Economy Review, 88, 1232-48.

[29] Savaskan, R.C., S. Bhattacharya and L.N. Van Wassenhove (2004), "Closed-Loop Supply Chain Models with Product Remanufacturing", Management Science, 50, 239-52.

[30] Simaan, M. and T. Takayama (1978), Game Theory Applied to Dynamic Duopoly Problems with Production Constraints", Automatica, 14, 16166.

[31] Slade, M.E. (1994), "What Does an Oligopoly Maximize?", Journal of Industrial Economics, 42, 45-61.

[32] Tirole, J. (1988), The Theory of Industrial Organization, Cambridge, MA, MIT Press. 
[33] Williamson, O.E. (1971), "The Vertical Integration of Production: Market Failure Considerations", American Economic Review, 61, 112-23.

[34] Williamson, O. (1975), Markets and Hierarchies: Analysis and Antitrust Implications, New York, Free Press.

[35] Zaccour, G. (2008), "On the Coordination of Dynamic Marketing Channels and Two-Part Tariffs", Automatica, 44, 1233-39.

[36] Zusman, P. and M. Etgar (1981), "The Marketing Channel as an Equilibrium Set of Contracts", Management Science, 27, 284-302.

[37] Yue, J.F., J. Austin, M.C. Wang and Z.M. Huang (2006), "Coordination of Cooperative Advertising in a Two-Level Supply Chain when Manufacturer Offers Discount", European Journal of Operational Research, $168,65-85$. 


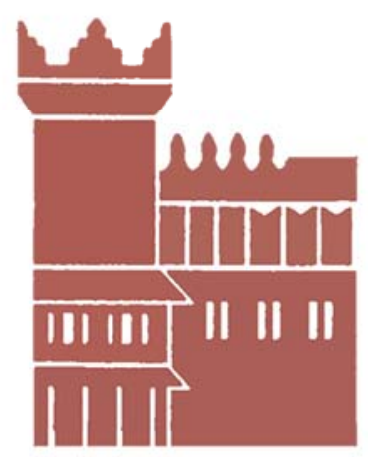

Alma Mater Studiorum - Università di Bologna DEPARTMENT OF ECONOMICS

Strada Maggiore 45

40125 Bologna - Italy

Tel. +39051 2092604

Fax +390512092664

http://www.dse.unibo.it 